\title{
Die Serumtherapie und Serumprophylaxis der akuten Infektionskrankheiten.
}

Klinischer Vortrag.

Von W. Kolle in Bern.

Wenn eiń Bakteriologe und Immunitätsforscher heutzutage für Kliniker oder wissenschaftlich denkende Aerzte auf wenigen Seiten einen Abriß der Serumtherapie der akuten Infektionskrankheiten geben will, ohne allzuviel jedem Bekanntes oder oft Gesagtes zu schreiben, so kann es nur geschehen durch Kennzeichnung der schwebenden Fragen, der noch zu lösenden Probleme unter Darlegung der allgemeinen Gesichtspunkte und neuen Methoden. Dieser letztgenannte Umstand bedingt aber, da viele Spezialkenntnisse des Fachbakteriologen für das Verständnis vorausgesetzt werden müssen, für die Darstellung die Benutzung von Beispielen einzelner Krankheiten, bei denen die Serumtherapie eine Rolle spielt. So lassen sich theoretische und praktische Gesichtspunkte vereinigen und gleichzeitig die Ergebnisse des Tierversuchs mit den therapeutischen Erfahrungen am Menschen ohne Ermüdung mitteilen. Dieser Weg soll deshalb bei den folgenden Mitteilungen beschritten werden. Zuvor noch einige wenige geschichtliche Bemerkungen.

Kaum 20 Jahre sind verflossen, seit die Serumtherapie wissenschaftlich begründet wurde. Es kann als allgemein bekannt vorausgesetzt werden, daß es E. v. Behring, welcher damals als Assistent zum Institut für Infektionskrankheiten kommandiert war, gelang, ein durch Immunisierung von Tieren gewonnenes Gegengift des Diphtheriegiftes, das Diphtherieantitoxin, mittels biologischer Methoden aufzufinden. Es ist sicher kein Zufall gewesen, dab diese später für die wissenschaftliche und praktische Medizin so bedeutungsvolle Entdeckung im Institut und gewissermaßen unter der Aegide von Robert Koch, auf dessen Veranlassung sich Behring, C. Fränkel und Brieger Ende der achtziger Jahre mit Studien der Diphtherieimmunität beschäftigten, gemacht worden ist. War doch das Institut des Begründers der modernen Bakteriologie, des Entdeckers der festen Nährböden und vieler spezifischer Infektionserreger stets eine Pflanzstätte für zielbewuß̣te Bestrebungen, namentlich auf dem Gebiete der Immunitätslehre und Heilung bzw. Verhütung der Infektions: krankheiten gewesen. Der Geist, welcher von Robert Koch auf die experimentellen Studien seiner Assistenten und Schüler im allgemeinen und auf die Immunisierungsversuche im besonderen einwirkte, konnte auch auf die Behringschen Arbeiten nicht ohne Einflaß bleiben.

Eine Vorbedingung für die Entdeckung der Antitoxine war die Auffindung der Bakterientoxine, im besonderen des Diphtherie- und Tetanusgiftes. Nur wenige Jahre nach der Auffindung des Diphtherietoxins durch Roux, Yersin, und unabhängig von diesen Forschern, durch Lo effler, und nach der unter Kochs Leitung von Kitasato ausgearbeitten Methode der Herstellung des löslichen Tetanustoxins wurden die Antitoxine durch Behring entdeckt. Ihre erste therapeutische Verwendung beim Menschen fanden die Antitoxine, im besonderen das Diphtherieantitoxin, in der damals unter Briegers Leitung stehenden Krankenabteilung des Institutes für Infektionskrankheiten seitens Kossels und namentlich gleichzeitig damit in der von Bagin sky geleiteten Kinderklinik des Kaiser Friedrich-Krankenhauses in Berlin, sowie in der Klinik von Heubner. Zwar wăren anfangs nur wenig wirksame Präparate von Behring hergestellt; enthielten doch die ersten von ihm für die Behandlung diphtheriekranker Kinder empfohlenen Diphtheriesera höchstens zehn und oft noch weniger Immunitäts-Einheiten im Kubikcentimeter. Es war namentlich Robert Koch, der vor der praktischen Verwendung dieser geringwertigen Serumpräparate, die, wie wir heute wisssen, nicht wirksam sein können, warnte und mit darauf drang, höherwertige Serumpräparate herzustellen. Dem klaren Blick Ehrlichs war es vorbehalten, die Methode systematischer Gewinnung von hochwertigen Diphtherieantitoxinen zu finden und, Hand in Hand damit gehend, exakte Verfahren der Wertbestimmung der Antitoxine in sinnreichster Weise zu erdenken. Wie groh.der Anteil Ehrlichs an dem jetzt Erreichten ist, kann - das sei in diesem kurzen historischen Ueberblick nebenbei erwähnt - am besten aus seinen Arbeiten ersehen werden, besonders 
übersichtlich an der Hand der ausgezeichneten Monographie von R. Otto. ${ }^{1}$ ) Welche bleibenden Verdienste sich Ehrlich auch auf anderen Gebieten der Immunitätslehre und allgemeinen Pathologie durch seine heuristisch so wertvolle Seitenkettentheorie erworben hat, ist bereits in dem vorhergehenden Artikel von Wassermann näher begründet.

Die für das Diphtherietoxin und -Antitoxin in experimenteller Arbeit zahlreicher Forscher, von denen Knorr und Wernicke nicht ungenannt bleiben dürfen, festgestellten Tatsachen wurden auch beim Tetanustoxin und -Antitoxin gefunden und erweitert.

Es bedurfte von der Einführung der antitoxischen Serumpräparate in die medizinische Praxis ab nur einer kurzen Spanne Zeit, um den Kliniken ein abschließendes Urteil über die therapeutische Verwendung der Antitoxine zu ermöglichen. Dies Urteil, wie es auf Grund zahlreicher Veröffentlichungen, klinischer Studien und Einzelerfahrung hervorragender Kliniker und Aerzte sich im Laufe der Zeit als feststehend jetzt gebilder hat, kann dahin zusammengefaßt werden, daß wir in dem Diphtherieantitoxin ein wirksames Heilmittel für die Diphtherieerkrankungen besitzen. Auch die Statistiken, in den verschiedensten Ländern gewonnen, geben deutliche Beweise für die Wirksamkeit des Präparates, indem sie die Herabsetzung der Mortalität zahlenmäßig nachweisen. Die Zahl derjenigen Aerzte, welche das Diphtherieantitoxin nicht anwenden oder ihm seine Wirksamkeit absprechen, wird von Jahr zu Jahr kleiner. Es läßt sich die Tatsache, daß eine kleine Minderzahl von Aerzten noch immer an dieser verneinenden Auffassung festhält, nur dadurch erklären, daß das Diphtherieserum auch bei frühzeitiger Anwendung in einem allerdings kleinen Prozentsatz der Diphtheriefälle im Stiche läßt, und hier kommen wir auf eines von den Problemen, die bei den antitoxischen Serumpräparaten und auch beim Diphtherieserum noch der Lösung harren. Denn es wäre verkehrt, jetzt schon die Behauptung aufzustellen, daß die Frage der spezifischen Therapie der Diphtherie, wie sie mit dem Antitoxin eingeleitet ist, etwa völlig gelöst und erschöpft wäre. Es mụ vielmehr Sache der Bakteriologen und Kliniker bleiben, weiter experimentell der Frage näherzuttreten. Von den Problemen, die noch geklärt werden müssen, will ich nur einige kurz erwähnen:

Warum versagt bei einem Prozentsatz der Diphtheriefälle das Diphtherieantitoxin, selbst frühzeitig und in genügender Menge angewendet, sodaß die Krankheit ihren Verlauf nimmt und tödlich endigt, trotzdem ein Ueberschub von Antitoxin im Blute kreist? Vielfach ist die Ursache für den Fehlschlag sicher in gleichzeitiger Mischinfektion zu suchen; der Tod erfolgt nicht durch das Diphtheriegift und seine für die Herznerven und die Organe deletären Wirkungen, sondern durch die gleichzeitig mit dell Diphtheriebacillen und auf dem Boden der von ihnen gesetzten Veränderungen eingedrungenen und reichlich vermehrten Streptococcen, bzw. Staphylococcen. Ein Prozentsatz der trotz Serumtherapie erfolgten Todesfälle ist ferner auf anderweitige anatomische Veränderungen in den Organen der Kranken zurückzuführen, z. B. auf Herzfehler und Nierenkrankheiten. Es handelt sich auch vielfach um schwächliche, wenig widerstandsfähige Individuen. Aber in einzelnen Fällen läßt das Serun tatstächlich im Stich aus Ursachen, die wir noch nicht kennen. Es mub nun das Bestreben der weiteren Forschung sein, die Ursachen dieser Fehlschläge aufzudecken und zugleich Wege zu weisen, mittels deren das Diphtherieserum noch wirksamer gemacht werden kann.

Die für ambozeptorenhaltige Sera so wichtige Frage der Polyvalenz spielt bei den antitoxischen Serumpräparaten, augenscheinlich keine Rolle. Ein Angriffspunkt für die Verbesserung des Diphtherieserums ist vielleicht in den Studien über das mit den Bakterienleibern hergestellte Diphtherieserum zu suchen. Dies durch die Untersuchungen von Martin, sowie von Lubow ski und später von Wass e rma nn bekannt gewordene Serumpräparat hat stark für Diphtheriebacillen agglutinierende, bzw. präzipitierende Eigenschaften. Es ist praktisch als trockenes Pulver in weitem Umfange schon ange-

1) Die staatliche Prüfung der Heilsera. Jena 1906, Gustav Fischer. wandt worden, um die nach dem Ablaufe und während der Krankheit im Munde, auf den Mandeln und im Rachen vorhandenen, aus den Krankheitsprodukten abgestoßenen Diphtheriebakterien zur Agglutination und damit zur rascheren Entfernung mittels desinfizierender Flüssigkeiten, Mundspülungen etc., geeigneter zu machen. Alle bisherigeǹ Erfahrungen über die mit Leibern anderer Bakterien hergestellten Sera sprechen aber dafür, dab dem agglutinierenden Diphtherieserum auch bakterizide Eigenschaften innewohnen. Es ist deshalb bereits mehrfach angeregt worden, das Präparat nicht nur als Schutzmittel zur Verhütung der Infektion in ähnlicher Weise wie das Antitoxin, welches eine passive Immunität von mehrwöchiger Dauer mit großer Zuverlässigkeit verleiht, heranzuziehen, sondern es auch beim diphtherieinfizierten Menschen, in dessen Gewebe die Diphtheriebacillen ja nicht nur Gift erzeugen, sondern sich auch noch vermehren, zur raschen $\mathrm{Ab}$ tötung der Bacillen zusammen mit dem Antitoxin zu injizieren. Es kann dem Verlangen nach Anwendung eines bakteriziden Diphtherieserums, zusammen mit dem antitoxischen Präparate, um so weniger die Berechtigung abgesprochen werden, als ein solches Serum ja unter keinen Umständen schädlich wirken kann. Denn die Diphtheriebacillenleiber sind verhältnismäßig wenig toxisch, und ein bakterizides bzw. agglutinierendes Serum, das zu einer Schädigung oder gar. Auflösung der Diphtheriebakterien führte, wird eine vermehrte Giftzufuhr beim diphtheriekranken Kinde nicht herbeiführen.

Wenn wir schon bei dem Diphtherieserum auf Grund der Erfahrung in der Praxis, soweit wir es als Heilmittel im Auge haben, und wegen der gelegentlich dabei vorkommenden Fehlschläge, noch weiterer experimenteller Forschung bedürfen, so ist das in noch weit größerem Umfange bei dem Tetanusantitoxin der Fall. Das Tetanusserum spielt namentlich in der Tiermedizin heutzutage eine große Rolle als Prophylaktikum. Wir besitzen in ihm ein Mittel, durch welches wir den Tetanus der Tiere, wie er sich häufig nach größeren Operationen, z. B. der Kastration, einzustellen pflegt, verhüten können. Die Schutzwirkung des Antitoxins beim Menschen ist, vorausgesetzt, daß eine genügend große Dosis von Antitoxin-Einheiten injiziert wird, eine ziemlich sichere. Seitdem bei den durch Schuß mit Platzpatronen verletzten Soldaten, die früher fast regelmäßig an Tetanus erkrankten, in jedem Falle die prophylaktische Seruminjektion unmittelbar nach der Verletzung angewandt wird, ist die Zahl der Tetanus-Erkrankungen und -Todesfälle in der Armee erheblich zurückgegangen. Aber wie in der Tiermedizin, so ist auch beim Menschen die therapeutis che Wirkung des Serums offenbar eine unsichere, selbst wenn große Mengen von Serum subcutan oder gar intravenös einverleibt werden. Wir können uns gut nach der Ehrlichschen Theorie das Versagen des Tetanusserums erklären, wenn wir mit Ehrlich annehmen, daß beim Ausbruch der ersten Tetanussymptome meistens schon so viel Gift an den Nervenzentren und in den Nervenbahnen verankert ist, dub eine Neutralisierung des im Blute und in den Gewebs. säften kreisenden Toxins durch das Antitoxin meist nutzlos bleibt. Man hat nun neuerdings versucht, durch direkte Einverleibung des Giftes in die regionären Nerven, z. B. beim Sitz der Tetanusinfektion an den Händen durch Einspritzung des Giftes in den Plexus brachialis, die Toxinwirkung zu paralysieren; man ging hierbei von der Annahme aus, dal das zlnächst im Blute kreisende Gift von den Endigungen der Nervenfasern aufgenommen und auf dem Wege der Neuronen nach den Zellen des zentralen Nervensystems hingeleitet wird. Bis jetzt haben indessen weder die intraneurale noch die intracerebrale, subdurale oder intralumbale Einverleibung des Antitoxins diejenigen therapeutischen Erfolge zu verzeichnen, die man theoretisch von dieser Anwendung des antitoxischen Serumpräparats erwarten sollte. Und die klinische Erfahrung steht vollkommen im Einklang mit den Ergebnissen des Tierversuches. Auch hier gelingt es nur eine verhältnismäßig kurze Zeit nach Einverleibung des Giftes bzw. des Infektionsstoffes, wie Dönitz zeigte, den tödlichen Ausgang der Intoxikation abzuwenden. Es öffnet sich also der Forschung noch ein ziemlich weites Feld, um diejenigen therapentischen Erfolge in der Praxis zu erzielen, die man auf Grund der Tierversuche, bei denen 
Toxin-Antitoxin-Gemische einverleibt werden, theoretisch logischerweise erwarten sollte. Bei einigen Fällen von menschlichem und tierischem Tetanus ist das Antitoxin von günstiger Wirkung gewesen, und die Serumtherapie sollte keinesfalls neben den sonstigen therapeutischen Maßnahmen vernachlässigt werden, schon deshalb nicht, weil die Serumtherapie auch bei dieser Krankheit unschädlich genannt werden kann.

Vielleicht ebenso große Verbreitung, wie die oben genannten, mit Bakterientoxinen hergestellten Antitoxine haben in den tropischen Ländern die mit tierischen Toxinen, den Schlangengiften gewonnenen antitoxischen Serumpräparate gefunden. Wenn das Gegengift nur frühzeitig genug eingespritzt wird, so ist es - nach zahlreichen Berichten kann daran nicht gezweifelt werden - ein zuverlässiges Heilmittel. Die praktische Schwierigkeit bei diesen Antitoxinen besteht darin, daß die Gifte verschiedener Schlangen untereinander verschieden sind, sodaß auch ein Antitoxin nicht für alle Gifte paft. Man muß deshalb in den verschiedenen Ländern mehrere Antitoxine - da es ja fast überall, wo überhaupt Giftschlangen vorkommen, mehrere Arten gibt - herstellen oder polyvalente Schlangengift-Sera.

Wenn wir schon bei den wohl am meisten und längsten studierten Antitoxinen keineswegs am Ende der experimentellen Forschung stehen, so gilt das noch in viel höherem Maße von anderen therapeutisch angewandten Serumpräparaten. Es gibt kaum eine Krankheit des Menschen oder Tieres, bei der nicht die Serumtherapie in irgend einer Form zur Verwendung vorgeschlagen wäre. Aus der großen Zahl der Serumpräparate haben sich eigentlich nur die folgenden erhalten und werden augenblicklich mit mehr oder minder grobem Erfolge von Aerzten und Klinikern angewandt: Das Dysenterieserum, Streptococcenserum, Pestserum, Meningococcenserum und Pneumococcenserum (für die Therapie des Ulcus corneae). Auberdem sind noch einige akut verlaufende Tierkrankheiten zu nennen, bei denen die Serumtherapie oder die Schutzimpfung mit Hilfe des Serums, bzw. in Kombination von Serum und Infektionserregern Anwendung und zum Teil weite Verbreitung gefunden hat: Rinderpest, Schweineseuche und Schweinerotlauf. Ehe an Beispielen die Wirkungsweise der einzelnen Serumpräparate, ihre praktische Brauchbarkeit und der Wert derselben als Schutzpräparate, bzw. Schutzimpfungsmittel beleuchtet werden, müssen wir einige allgemeine Bemerkungen über die spezifischen Stoffe der Immunsera und ihren Nachweis vorausschicken. Alle Serumpräparate, von denen hier die Rede sein soll, sind spezifis ch in ihrer Wirkung, d. h. sie beeinflussen nur diejenigen Krankheiten bzw. deren Infektionserreger, mit welchen sie hergestellt sind. Es ist aber gleichgültig, welche Methode man anwendet, um die Spezifi zität nachzuweisen. Die Wirksamkeit beruht auf der Anwesenheit von spezifischen Stoffen, von denen wir bis jetzt vier voneinander verschiedene kennen gelernt haben. Es sind dies die Bacteriolysine R. Pfeiffers, die Agglutinine, die Prä zipitine und die opsonine, über deren Bedeutung für die Immunitätslehre und die Praxis in dem vorhergehenden Aufsatze von Wassermann das Nötige mitgeteilt ist.

Nicht alle Serumpräparate lassen sich in die eine oder andere Klasse einteilen, je nachdem sie Bakteriolysine oder Präzipitine oder Opsonine enthalten, schon deshalb nicht, weil meistens mehrere dieser Körper nebeneinander in dem Immunserum vorhanden sind. Wir sind sogar über die Art der Wirksamkeit verschiedener Serumpräparate noch keineswegs vollkommen orientiert, trotz des Nachweises des einen oder anderen dieser Stoffe in ihnen. Es empfiehlt sich deshalb, die Bezeichnung: Antiinfektiöse Serumpräparate allgemein für die nicht vorwiegend antitoxischen Schutz- und Heilsera zu benutzen. Diese Bezeichnung bringt zum Ausdruck, daß sich die Wirkung der Serumpräparate gegen die lebenden Infektionsstoffe richtet, und läßt es offen, wie diese Wirkung im einzelnen zustande kommt. Ich werde im folgenden noch mehrfach begründen, warum die strenge schematische Klassifizierung der Serumpräparate verfrüht ist.

Bezüglich der mit Choleravibrionen und Typhusbakterien hergestellten bakteriziden Serumpräparate können wir an dem zuerst von R. Pfeiffer vertretenen Standpunkte festhalten. daß es im wesentlichen die Endotoxine, also die in den Bakterienleibern enthaltenen Gifte sind, welche bei diesen Krankheiten einerseits die schweren Symptome, Fieber etc., und anderseits die Bildung der Bakteriolysine auslösen. Es wird von manchen Autoren allerdings noch daran festgehalten, daß man unter Umständen neben diesen reichlich in den mit Bakterienleibern hergestellten Serumpräparaten vorhandenen Bakteriolysinen auch A n ti - Endo tox in e, also Gegengifte gegen die Gifte der Bakterienleiber erhalten kann. Die neuerlichen Angaben von Mac Fadyan, daß es ihm gelungen sei, durch Zertrümmerung der bei ganz niedrigen Temperaturen gefrorenen Cholerabakterien - $-180^{\circ} \mathrm{C}$ - ein lösliches Choleragift und mit Hilfe desselben bei Tieren ein Antitoxin herzustellen, bedürfen zwar noch der Nachprüfung, lassen aber doch schon so viel erkennen, daf MacFadyan kaum etwas anderes in den Händen gehabt haben dürtte als Endotoxine und daß dementsprechend sein Serum vielleicht wesentlich antitoxische Wirkungen gegen die Endotoxine besaß. Auch 0 tto, der Mitarbeiter Ehrlichs, läßt die Annahme zu, daß alle antibakteriellen Sera eine gewisse Quote Anti-Endotoxin enthalten. Aehnlich liegen die Verhältnisse nach den Arbeiten von Besredka auch für das Typhusserum. Soviel läßt sich, trotzdem die Frage der Gewinnung von Anti-Endotoxinen noch nicht ganz abgeschlossen ist, sagen, daf mit den bisherigen Methoden sich keine Anti-Endotoxine herstellen lassen, für welche Ehrlichs Gesetz der Multipla wie für die echten Antitoxine, gilt.

Ganz zu trennen von dieser Frage, Anti-Endotoxine zu erzeugen, ist diejenige des Nachweises löslicher sezernierter Gifte bei Typhus und speziell Cholera und der Gewinnung von echten Typhus- und Choleraantitoxinen. Diese Frage ist neuerdings wieder aktuell geworden durch die Angaben von R. Kraus, daf die bekannten El-Tor-Vibrionen, welche von F. Gotschlich isoliert und deren Cholerakultur von E. Got. schlich und mir zuerst experimentell nachgewiesen wurde ein lösliches Toxin produzieren. R. Kraus hat an Tieren mit dem Toxin der El-Tor-Vibrionen ein Antitoxin hergestellt und bezeichnet dieses als ein Choleraantitoxin. Auch diese Angaben von $\mathrm{K}$ raus, der übrigens die Choleranatur der El-Tor-Vibrionen, wie mir scheint, in nicht ganz konsequenter Weise mit Rücksicht auf die mitgeteilten Versuche bestreitet, bedürfen noch weiterer Klärung und Nachprüfung, ehe man von einem Choleraantitoxin sprechen kann. Bereits früher glaubten ja schon Metschnik off, Roux und Salimbeni sowie Behring, ein lösliches Choleragift, hergestellt mittels der bekannten Kollodiumsackmethode, in Händen gehabt zu haben. Es ist inzwischen nichts über die therapeutische Wirksamkeit oder praktische Verwendung eines derartigen Serums wieder laut geworden. Nach allem, was wir über die Entstehung des Choleraprozesses wissen, der ja im wesentlichen als ein Infektionsprozeß des Dünndarmepithels mit nachfolgender Vergiftung durch die Endotoxine der Choleravibrionen aufgefaßt werden mub, erscheint es nicht sehr aussichtsvoll, nach einem supponierten sezernierten Choleratoxin zu suchen und auf dem Wege einer antitoxischen Therapie bei dieser Krankheit viel zu erreichen.

Das gleiche, was hier für die Cholera gesagt ist, gilt auch für die Gewinnung des löslichen Typhustoxins, für die Erklärung der Vergiftungssymptome beim Typhus und die Aussichten einer antitoxischen Serumtherapie. Viel aussichtsvoller erscheint dagegen beim Typhus, da es sich ja im Gegensatz zu der Cholera um eine Infektion des Blutes mit Typhusbacillen und eine Vermehrung derselbeil in den verschiedensten Organen handelt, die therapeutische Anwendung eines hochwertig bakteriziden Typhusserums, zumal wenn es durch Variation der Methoden gelänge, die Anti-Endotoxine in stärkerer Konzentration in dem Serum der Immunisierungstiere zu gewinnen. Die Aengstlichkeit, mit der man das hochwertig bakterizide Typhusserum, das nebenbei auch hochwertig agglutinierend wirkt, in der Therapie beim typhuskranken Menschen anzuwenden sich bisher gesträubt hat, erscheint mir nicht zu sehr berechtigt. Denn die Furcht, daf durch Anwendung eines bakterienauflösenden Serums eine Giftüberlastung des Körpers durch die dann freiwerdenden Endotoxine herbeigeführt werden könnte, sollte schon auf 
Grund der Erfahrungen des Tierversuches nicht abhalten, in geeigneten Fällen beim Typhus das hochwertig bakterizide Serum in Form von kleineren, öfter wiederholten Serumgaben zu versuchen. Bietet sich doch für den Therapeuten der Vorteil, durch Einverleibung des bakterienfeindlichen Serums die weitere Vermehrung der Typhusbakterien im Blute oder in den Organen zu beschränken.

Bei der Dysenterie sind die experimentellen Forscher glücklicher gewesen, soweit es sich um die Erreichung praktischer Ergebnisse handelt. Wenngleich das Urteil über die Wirkung des Dysenterieserums noch nicht als ein so abgeschlossenes betrachtet werden kann, wie wir es bezüglich des Diphtherieserums uns haben bilden können, so kann man doch schon jetzt behaupten, daß das Dysenterieserum tatsächlich als ein therapeutisch wirksames Mittel sich Eingang in die Klinik verschafft hat. Zum Verständnis der verschiedenen, in der Praxis benutzten Serumpräparate sei mit einigen Worten auf die Pathogenese der epidemischen Dysenterie hingewiesen. Die Ursache der epidemischen Ruhr, der weitverbreitetsten Form dieser Infektionskrankheit, ist der Shiga-Krusesche Bacillus, welcher sich in der Schleimhaut des Dickdarmes ansiedelt und dort in dreifacher Weise durch Gifte wirkt: durch Erzeugung einer schweren hämorrhagischen Entzündung, zweitens durch Nekrotisierung des Epithels und endlich durch die allgemein wirkenden Giftstoffe, welche den Tod zusammen mit der durch Nahrungsmangel bedingten Schwäche herbeiführen. Die neueren Forschungen haben ergeben, daß es sich höchstwahrseheinlich nicht nur um die Wirkungen von Endotoxinen der Dysenteriebakterien handelt, sondern dab auch lösliche Gifte von dieser Bakterienart erzeugt werden.

Diese löslichen, von Shiga, Rosenthal und neuerdings von Kraus und Dörr, sowie Dopter und Vaillard in 14-18tägigen Bouillonkulturen nachgewiesenen Dysenterietoxine lassen sich am besten durch den Versuch an Kaninchen demonstrieren. Man kann sich ganz, bezüglich der Gifte, dem jüngst von Kruse vertretenen Standpunkte anschließen, welchen dieser um die Erforschung der Aetiologie der Ruhr neben Shiga verdiente Autor vertritt, nämlich „die Verallgemeinerungen beiseite zu lassen und je nach dem einzelnen Fall von lichtempfindlichen und lichtbeständigen, immunisierenden und nichtimmunisierenden, schwer oder leicht sich von ihren Bakterien trennenden, schnell oder langsam gebildeten spezifischen oder nichtspezifischen Giften zu sprechen". Wir stehen, zumal die Chemie hier im Stich läßt, noch im Anfang der Erforschung der Gifte, deren Reindarstellung, wie bekanntlich aus den Forschungen Briegers hervorgeht, noch ein pium Desiderium ist. Aehnlich unvollkommen sind unsere Vorstellungen und Kenntnisse über das Spektrum der mi Bakterienleibern hergestellten Sera. Je nachdem man nun die Immunisierung von größeren, zur Serumherstellung benutzten Tieren mehr mit den die Endotoxine enthaltenden Bakterien in Form der Agarkulturen der Dysenteriebakterien vornimmt, oder mit den lösliche Toxine enthaltenden Filtraten von Bouillonkulturen ausführt, erhält man im ersteren Falle mehr bakterizid wirkende und Anti-Endotoxine enthaltende, im letzteren Falle vorwiegend die Toxine paralysierende Serumpräparate. Wichtig für die theoretische Auffassung der Immunisierungsvorgänge und der Reaktion des Tierkörpers auf die Einverleibung der Antigene ist die am kranken Menschen gewonnene Tatsache, daß mit beiden Arten der Dysenteriesera gute therapeutische Erfolge bei der Behandlung erzielt worden sind, mit den ersteren von Shiga und Kruse, mit den letzteren von Dopter und Vaillard, sowie von Kraus und Dörr, und beide Arten des Dysenterieserums entfalten beim Menschen auch eine sichere Schutzwirkung. Die Prüfung der Serumpräparate erfolgt an Kaninchen. Es läßt sich bei dieser Tierart nicht nur die Schutzwirkung des Serums gegenüber einer später erfolgenden Infektion, bzw. gegen die Intoxikation der Dysenteriebacillen erzielen, sondern auch nach erfolgter Infektion oder Intoxikation das Leben der Tiere durch nachfolgende Seruminjektion erhalten. Es dürfte sich empfehlen, in Zukunft beide Arten von Dysenterieserum kombiniert anzuwenden, das vorwiegend bakterizide, mit Bakterienleibern gewonnene, welches auch eine erhebliche Quote Antiendotoxine enthält, um ein Fortschreiten der Infektion zu verhindern, das mit Filtraten hergestellte, vorwiegend antitoxische, um die schon gebildeten sezernierten Toxine zu paralysieren.

Bei dem Streptococcenserum, das weitgehende therapeutische Verwendung bei den verschiedensten StreptococcenAffektionen gefunden und sich bei vielen Aerzten als ein beachtenswertes Heilmittel eingebürgert hat, sind noch manche streitige Punkte experimentell zu klären. Sicher ist, daß das Streptococcenserum als antitoxisch nicht bezeichnet werden kann, schon deshalb nicht, weil wir lösliche Streptococcengifte noch nicht herstellen und deshalb bei der Serumgewinnung nicht benutzen können. Bei allen Methoden, welche zur Herstellung des Serums benutzt werden, gelangen vielmehr zunächst abgetötete Agar- oder Bouillon-Kulturen und dann lebende Kulturen der gleichen Art, die ein Alter von 24 bis 48 Stunden haben, zur Verwendung. Es werden jetzt in der Praxis hauptsächlich Sera angewandt, die nach den von Marmorek, Aronson oder Tavel angegebenen Verfahren hergestellt sind. Die Hauptunterschiede der einzelnen Präparate bestehen darin, daf entweder ein durch zahlreiche Kaninchenpassagen für Tiere sehr virulent gemachter Streptococcenstamm (Marmorek) oder daß (Tavel) möglichst viele, aus den verschiedensten Streptococcen-Krankheitsprozessen gezüchtete Kulturen benutzt werden. Es handelt sich bei den ersten beiden Sera um monovalente, bei dem an zweiter Stelle genannten aber um die sogenannten poly valenten Streptococcensera, die namentlich Tavel wissenschaftlich vertreten hat Die Kulturen werden nach Tavels Vorgang ohne Tierpassage weiter gezüchtet. Einen Mittelweg hat A r o n s on eingeschlagen damit, daß er teils Pferde mit virulenten Passagestämmen und teils andere mit avirulenten menschlichen Originalstämmen immunisiert und dann die Sera der verschiedenen Pferde mischt. Neuerdings hat Ruppel dies Verfahren noch etwas verbessert dadurch, daß er gleichzeitig jedes mit den Originalstämmen immunisierte Pferd zugleich mit einer virulenten Passagekultur einspritzt, sodaß also die von der letzteren erzeugten Antikörper als Indikator für den Gehalt des Serums an Immunitätseinheiten dienen. Es fehlt bei dieser Methodik aber noch der Nachweis, daß die Antikörper erzeugende Fähigkeit der tierpathogenen und avirulenten Kulturen eine gleiche ist.

Nach den bisherigen therapeutischen Erfahrungen dürfte den polyvalenten Streptococcenpräparaten unbedingt der Vorzug vor den monovalenten zu geben sein, denn die immunisatorischen Differenzen der einzelnen Streptococcenstämme sind ganz evident und konstant. Es läßt sich experimentell immer wieder zcigen, daß ein mit einem Stamm hergestelltes Serum meist nur gegen diesen einen Stamm und eine kleine Anzahl anderer Stämme im Tierversuch schützt, nicht dagegen alle Stämme beeinflußt. Nach W assermann sind diese immunisatorischen Unterschiede im Sinne der Ehr lichschen Theorie auf Unterschiede im Bau des Rezeptorenapparates der einzelnen Streptococcenstämme zurückzuführen. Die Polyvalenz ist nicht in dem Sinne aufzufassen, als ob die aus verschiedenen Streptococcen-Krankheitsprozessen isolierten Streptococcenstämme sich immunisatorisch verschieden verhielten. Das ist keinesfalls immer notwendig. Ja, in dieser Beziehung ist eine Polyvalenz des Serums nach unserer heutigen unitarischen Auffassung $\mathrm{der}$ bei den verschiedenen Krankheitsprozessen (Erysipel, AbsceB, Angina, Sepsis, Wundeiterung, Puerperalinfektionen etc.) vorkommenden und diese bedingenden Streptococcenstämme als einer einzigen Spezies nicht mehr aufrecht $\mathrm{zu}$ erhalten. Ein in diesem Sinne polyvalentes Serum wurde schon vor längerer Zeit von Denys und van der Velde empfohlen. Von diesen Forschern wurden für die Immunisierung Streptococcenstämme aus möglichst allen Streptococcen-Krankheitsprozessen gewonnen und benutzt. Praktisch genommen, leistet das im Sinne von Denys und van der Velde polyvalente Serum annähernd dasselbe, wie das von Wassermann und Tavel empfohlene. Aber die theoretische Begründung der Auswahl so vieler verschiedener Stämme seitens der letztgenannten Autoren ist derjenigen von Denys vorzuziehen. Daß aber die Polyvalenz der Streptococcensera für die Praxis und Therapie von Bedeutung ist, das ergeben die von Denys, Aronson und Tavel, neuer- 
dings von Moser und Menzer ${ }^{1}$ ) gesammelten therapeutischen Erfolge beim kranken Menschen. Auch andere Autoren, namentlich Chirurgen, haben zum Teil recht günstige Erfahrungen mit diesem Serumpräparate gemacht bei akuten wie den chronischer verlaufenden Streptococceninfektionen. Ein statistischer Nachweis für die therapeutische Wirksamkeit des Streptococcenserums läßt sich allerdings aus den jedem Arzte bekannten Gründen nur außerordentlich schwer erbringen, schon deshalb, weil die Mortalität sich bei diesen Krankheitsprozessen schwer in Zahlen ausdrücken läßt und weil viele Erkrankungen auch ohne Serumanwendung einen kritischen Heilungsverlauf zeigen. Wenngleich nun das Streptococcenserum bei manchen Krankheitsfällen, teils weil schon zu starke Ueberschwemmung des Körpers mit Streptococcen und sekundäre Ansiedelung derselben in den verschiedensten Organen erfolgt ist, oder weil von abgekapselten Eiterherden aus dauernd grofe Mengen von Streptococcen ins Blut gelangen oder weil dieses Serum auf einzelne Streptococcenstämme immer ohne Wirkung bleibt, oder endlich aus Ursachen, die wir noch nicht kennen, im Stiche läßt, so sind doch viele Aerzte auf Grund eklatanter Erfolge Anhänger der Serumtherapie geworden. Es liegt hier also ähnlich wie beim Diphtherieserum, wo auch durch die Einzelbeobachtung erfahrener Aerzte die Wirksamkeit des Antitoxins in der Therapie früher und zum Teil bestimmter erkannt ist, als dies durch die Statistik, die übrigens bei der Diphtherie wohl heranzuziehen ist, bewiesen wurde.

Wenn wir uns nun fragen, worauf die Wirksamkeit des Streptococcenserums beruht, so ist diese Frage noch keineswegs ganz entschieden. Dab Antitoxine in demselben nicht vorhanden sind, scheint wohl sicherzustehen; auf die Anwesenheit von Bakteriolysinen und Opsoninen läßt sich aber die Wirksamkeit des Streptococcenserums allein nicht zurïckführen. Man wird auch hier am besten tun, ähnlich wie bei dem nun zu besprechenden Pestserum, die Wirksamkeit des Serums als eine anti-infektiöse im allgemeinen $\mathrm{zu}$ bezeichnen. Eine besondere Schwierigkeit macht die Prüfung des Streptococcenserums; der Tierversuch läßt deshälb vielfach im Stich, weil die monovalenten Sera ja nur gegen eine Minderzahl von Streptococcenstämmen schützen und man deshalb ein für einzelne Stämme hochwertiges Präparat nicht ohne weiteres als allgemein hochwertig bezeichnen kann. Die polyvalenten Sera aber lassen trotz größerer Breite der Wirksamkeit, gegenüber den hochvirulenten, zur Prüfung allein benutzbaren Stämmen, im Tierversuch häufig im Stich, selbst wenn man sie mit zur Immunisierung heranzieht. Das Rup pelsche Verfahren dürfte bezüglich der Wertbestimmung noch das am meisten zu empfehlende sein. Ueber die Schutzwirkung des Streptococcenserums ist noch wenig Sicheres bekannt. Seit die Antisepsis und Asepsis in der Chirurgie allgemein angewandt werden, hat die Verwendung eines schützenden Serums wenig Bedeutung.

(Schluß folgt.)

1) Das als sogenanntes Scharlachserum mit den bei Scarlatina-Ángina vorkommenden Streptococen hergestellte sowie das Serum, welches von. Menzer mit den als Mischinfektionserreger bei Gelenkrheumatismus gefundenen Streptococcen gewonnen wurde, sind nichts anderes als polyvalente Streptococcensera. Auch das bei der Therapie des Ulcus corneae von Römer empfohlene Pneumococcenserum gehört prinzipiell zu den polyvalenten Streptococcensera. 